\title{
Kernos
}

Revue internationale et pluridisciplinaire de religion grecque antique

$14 \mid 2001$

Varia

\section{Le jeu des épithètes dans les Hymnes orphiques}

\section{Marianne Hopman-Govers}

Édition électronique

DOI : $10.4000 /$ kernos.766

ISSN : 2034-7871

\section{Éditeur}

Centre international d'étude de la religion grecque antique

\section{Édition imprimée}

Date de publication : 1 janvier 2001

Pagination : 35-49

ISSN : 0776-3824

Référence électronique

Marianne Hopman-Govers, "Le jeu des épithètes dans les Hymnes orphiques », Kernos [En ligne],

14 | 2001, mis en ligne le 14 avril 2011, consulté le 01 mai 2019. URL : http://journals.openedition.org/ kernos/766 ; DOI : 10.4000/kernos.766 
Kernos, 14 (2001), p. 35-49.

\section{Le jeu des épithètes dans les Hymnes orphiques*}

Un trait remarquable du corpus des quatre-vingt sept hymnes qui nous ont été transmis sous le nom d'Orphée ${ }^{1}$ tient à l'accumulation d'épithètes qui constitue la structure de chaque hymne. Contrairement aux hymnes homériques, de Callimaque et de Proclus qui les accompagnent dans la plupart des manuscrits ${ }^{2}$, les Hymnes orpbiques sont pauvres en narration et constitués essentiellement d'adjectifs épithètes, participes ou courtes propositions relatives $^{3}$. Pour reprendre le schéma hymnique proposé par Carl Ausfeld

* Une première version de cet exposé a été présentée à l'occasion du colloque CorHaLi organisé par $\mathrm{M}$. Claude Calame sur le thème « La figure d'Orphée et les poèmes orphiques ". Je remercie de leurs suggestions les participants à cette rencontre, et notamment Mmes et MM. Fabienne Blaise, Claude Calame, Pierre Judet de la Combe, Stella Georgoudi, Richard Martin, Anne-France Morand, Gregory Nagy, Charles Segal et Froma Zeitlin. Je suis aussi très reconnaissante à Mme Vinciane Pirenne-Delforge pour les commentaires stimulants dont elle m'a fait part. Enfin je remercie tout particulièrement M. Albert Henrichs pour la grande générosité avec laquelle il m'a prodigué conseils, critiques et encouragements tout au long de la rédaction de cet article.

1 En l'attente de l'édition de A. Bernabé, le texte le plus récent des Hymnes orphiques est celui de G. Quandt, Orphei bymni, Berlin, $1962^{3}$, dont je suis la numérotation. Une concordance a été établie par A. BERNABÉ, Orpbei bymnorum concordantia, Olms, 1988, et nous disposons de la traduction anglaise de A. Atranassakis, The Orphic Hymns: Text, Translation and Notes, Missoula, 1977. Il n'existe pas pour l'instant de commentaire complet plus récent que celui de $M$. Koops, Observationes in bymnos Orpbicos, Leiden, 1932. En revanche, on trouve des commentaires sur certains hymnes de la collection dans R.L. Hunsucker, A Select Commentary on the Orpbic bymns, Diss. Princeton, 1973 (avec une bibliographie complète) et L.J. Alderink, "Orphic Hymn 13: to Kronos", in M. Kiley (éd.), Prayer from Alexander to Constantine, Londres, 1997, p. 190-194. Parmi les études sur les Hymnes, citons celles de L. vaN LIEMPT, De vocabulario bymnorum Orpbicorum atque aetate, Permereno, 1930; W.K.C. Guthrie, "Epithets in the Orphic Hymns", CR 44 (1933), p. 216-221; J. Rudhardr, "Quelques réflexions sur les hymnes orphiques", in Ph. Borgeaud (éd.), Orpbisme et Orpbée, Genève, 1991, p. 263-283; A.-F. Morand, "Orphic Gods and other Gods", in A.B. Lloyd (éd.), What is a God?, London, 1997, p. 169-181.

2 Sur cette tradition manuscrite : O. KERN, "Die Herkunft des Orphischen Hymnenbuches", in Genetbliakon für Carl Robert, 1910, p. 87 sq. et T. Gelzer, "Zum Codex Mosquensis und zur Sammlung der homerischen Hymnen", Hyperb. 1 (1994), p. 113-136.

3 On retrouve là le « Partizipialstil der Prädikation » et le « Relativstil der Prädikation » de l'étude classique d'E. NoRden, Agnostos Theos: Untersucbungen zur Formengescbichte Religiöser Rede, Stuttgart, 1956, p. 166-176. 
(invocatio - pars epica - precatio) $)^{4}$, la pars epica, si l'on entend par là un récit narratif des aretai du dieu, se trouve dans les Hymnes orpbiques réduite à la portion congrue.

L'accumulation d'épithètes ${ }^{5}$ n'est pas une technique propre aux Hymnes orpbiques. Il semble plutôt qu'il s'agisse d'une tendance inhérente au genre de l'hymne, que l'on trouve déjà en germe dans certains des hymnes homériques anciens ${ }^{6}$. Ces listes deviennent plus fréquentes à partir de l'époque alexandrine ${ }^{7}$. On les trouve aussi dans l'Hymne à Zeus de Cléanthe, les Hymnes à Isis transmis sous le nom d'Isidore et dans un hymne à Bacchus qu'Ovide met dans la bouche des femmes de Thèbes ${ }^{8}$. Enfin la technique est assez fréquente dans des textes d'époque impériale, comme certains papyrus magiques, quelques-uns des oracles d'Apollon à Claros et Didymes et les Dionysiaques de Nonnos. On trouve même dans l'Anthologie Palatine deux hymnes à Apollon et Dionysos composés uniquement d'épithètes arrangées par ordre alphabétique 9 . Pourtant, les Hymnes orpbiques ont ceci de particulier qu'ils poussent la technique à son point limite; comme l'a souligné Jean Rudhardt $^{10}$, ces textes tiennent essentiellement en un long développement de l'invocation initiale, suivi de l'énoncé de la demande de l'orant.

C'est cette particularité caractéristique et pourtant peu étudiée ${ }^{11}$ des Hymnes orphiques que je propose d'examiner, en considérant les épithètes d'abord sur le plan de leur sélection paradigmatique, puis de leur organisation syntagmatique. À côté des épithètes traditionnellement attachées à telle ou telle divinité, on verra qu'une grande partie des adjectifs choisis sont liés

4 C. AusFeld, De Graecorum precationibus quaestiones, Leipzig, 1903 (Jabrb. Class. Pbil., 28), p. 505-537.

5 Sur les listes d'épithètes, voir les remarques suggestives de A.-J. FEstugière, La révélation d'Hermès Trismégiste II, Paris, 1949, p. 313-316. (sur l'hymne de Cléanthe à Zeus) et L. RoBERT, "Un oracle gravé à Oinoanda", CRAI (1971), p. 610-619, repris dans OMS V (1989), p. 630-639. Pour une typologie des épithètes, voir A. Henrichs, "Despoina Kybele: ein Beitrag zur religiösen Namenkunde", HSPb. 80 (1976), p. 253-286 et F. Graf, "Namen von Göttern im klassischen Altertum", in E! Eichler et al. (éds), Ein internationales Handbuch zur Onomastik, Berlin/New York, 1996, p. 1823-1837.

6 Cf. $H H$ VI, 1 et VI, 19 (Aphrodite), XXII, 6 (Poseidon). A.-J. Festugik̀re a même suggéré d'utiliser les listes d'épithètes comme critère pour établir une datation relative des Hymnes bomérqques (o.c. [n. 5] p. 315, n. 3).

7 Cf. Théocrite, 1, 100-101 et 22, 135-136; Callimaque, Hymnes, 1, 91; 3, 269; 5, 43-44; Anth. Pal. VI, 10 (Antrpater). La tendance est suffisament répandue à l'époque alexandrine pour que l'on en trouve des caricatures, comme dans Antb. Pal. V, 135 (= Anon. 57 Gow-Page) « A un flacon».

8 Ov., Met. IV, 11-30.

9 Anth. Pal. IX, 524 (Dionysos) et 525 (Apollon).

10 RudHardi, l.c. (n. 1), p. 265.

11 Les études sur les. Hymnes orpbiques qui s'attachent le plus aux épithètes sont celles de Guthrie, l.c. (n. 1) et de Rudhardt, l.c. (n. 1). 
aux besoins de la prière finale ou illustrent quelques grands thèmes de glorification des dieux, qui traversent et unifient la collection. On examinera ensuite les effets produits par l'organisation de ces épithètes : parallèlement à des jeux musicaux sur les rythmes et les sonorités, la comparaison avec d'autres textes suggère de voir dans ces listes une forme extrême de concentration visant à décrire la nature de la divinité invoquée.

Un certain nombre des épithètes utilisées dans les Hymnes orpbiques proviennent de la tradition littéraire, en particulier de l'héritage homérique et

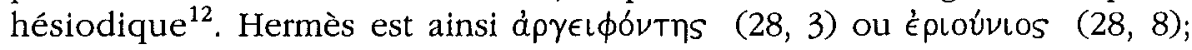

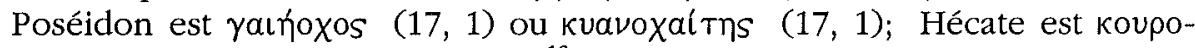
tpóфos $(1,8)$. Par ailleurs, Guthrie ${ }^{13}$ a montré que d'autres épithètes ne sont pas traditionnelles, mais font allusion à un épisode connu, dont elles présentent comme une narration condensée. Ainsi l'hymne 12 à Héraklès fait allu-

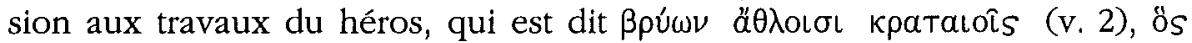

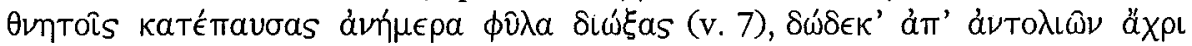
$\delta v \sigma \mu \hat{\omega} \nu \hat{a} \theta \lambda \alpha \delta \mathrm{l} \epsilon \rho \operatorname{\rho } \omega \nu$ (v. 12), sans pour autant en donner une narration complète. Par cette technique d'allusions, les Hymnes orpbiques se rapprochent des poètes alexandrins et en particulier de Callimaque ${ }^{14}$, qui sont parmi nos premiers exemples de juxtaposition d'épithètes.

Ce jeu avec l'héritage littéraire est cependant poursuivi de façon inégale dans le recueil. Si certains hymnes, tel celui à Poséidon (17), sont très proches thématiquement et lexicalement de l'héritage homérique, d'autres en revanche, comme l'hymne à Pan (11), s'écartent de ce modèle et offrent une série d'épithètes dont une seule (vóptos) est attestée dans le répertoire des épithètes littéraires dressé par Karl Bruchmann ${ }^{15}$. D'autre part, dans un même hymne, les épithètes traditionnelles voisinent souvent avec des hapax legomena ou des adjectifs communs à plusieurs divinités. Dans l'hymne 12 mentionné précédemment, l'image traditionnelle d'Héraklès est doublée d'une dimension cosmique, qui le rapproche d'autres divinités et l'assimile à Apollon, Hélios et Paean. Sans parler des divinités extérieures au panthéon traditionnel, telles Protogonos ou Physis, même les Olympiens reçoivent donc ici une présentation particulière, distincte de leur image littéraire et religieuse habituelle.

Une large partie des épithètes sont ainsi communes à plusieurs divinités, participant du syncrétisme caractéristique de ces textes. Héraklès est appelé

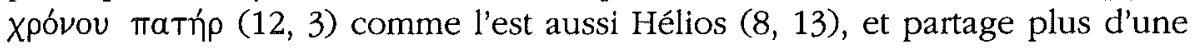

12 Cf. QUandT, o.c. (n. 1), et Koops, o.c. (n. 1), passim.

13 Guthrie, l.c. (n. 1).

14 Sur l'utilisation des épithètes dans les Hymnes de Callimaque, voir A. Henrichs, "Gods in Action: the Poetics of Divine Performance in the Hymns of Callimachus", in A. Harder (éd.), Callimacbus, Groningen, 1993 (Hellenistica Groningana, 1), p. 127-147.

15 K. BruchmanN, Epitheta deorum quae apud poetas Graecos leguntur, Leipzig, 1893. 
épithète avec Héphaïstos. On est frappé par exemple des ressemblances lexicales et rythmiques entre le vers 12,6 qui qualifie Héraklès, et le vers 66,5 , à propos d'Héphaïstos :

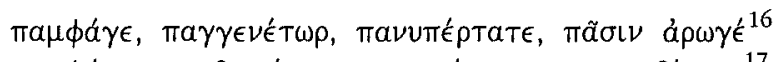

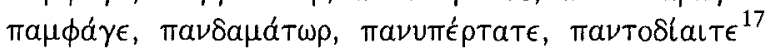

On pourrait multiplier les exemples d'épithètes communes, qui donnent au panthéon des Hymnes orpbiques son homogénéité.

De grands thèmes se dessinent ainsi et, sans en aucune façon prétendre à l'exclusivité, on peut évoquer quelques-uns de ces motifs par lesquels sont glorifiés les dieux des Hymnes orpbiques. Nombre d'épithètes s'attachent à l'ancrage spatial du dieu, le plus souvent pour insister sur l'omniprésence de son action. Un petit groupe d'hymnes du début du recueil fait exception puisque, peut-être sur le modèle stoïcien, les divinités s'y trouvent associées avec un élément particulier : Héra avec l'air et les vents (16, 1-4), Poséidon avec la mer (17), Pluton avec la terre (18). On retrouve donc ici le modèle homérique et hésiodique du partage des champs d'action des divinités (18, 6). Le plus souvent, cependant, les divinités présentées dans les Hymnes orphiques participent simultanément de tous les règnes. Hécate est dite

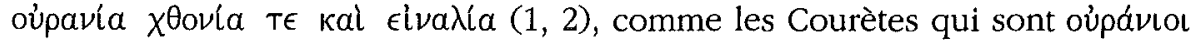

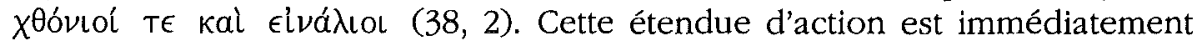
liée au polymorphisme et au syncrétisme qui traversent les Hymnes.

A la multiplicité des champs d'action s'ajoute la double appartenance

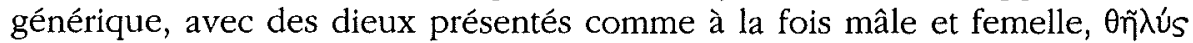
Tє kal ăponv (Sélènè 9, 4; Athéna 32, 10; Misè 42, 4). Ce motif n'est pas propre aux Hymnes orphiques; Platon y fait déjà allusion dans le Banquet :

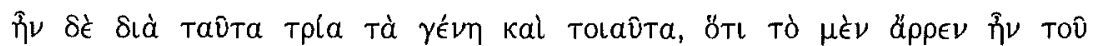

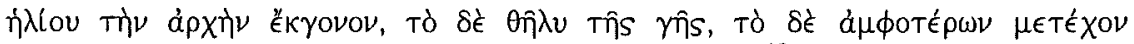

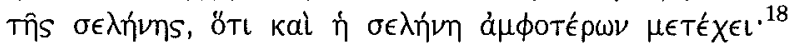

et on le retrouve dans les hymnes des papyrus magiques :

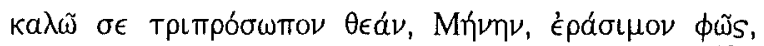

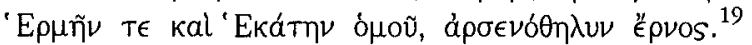

Diversité spatiale comme androgynie participent d'un même concept, omniprésent dans les Hymnes : celui de dieux polymorphes. Ce concept est largement reflété dans le lexique avec l'emploi d'adjectifs comme aiodó-

$16 O H, 12,6$,

$17 \mathrm{OH}, 66,5$

18 Platon, Symp., 190b.

19 PGM IV, 2610-2611 = Hymn., 19, 25-26 (Heitsch) 


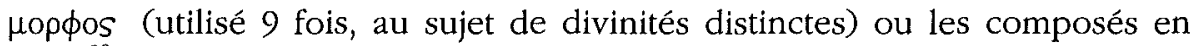

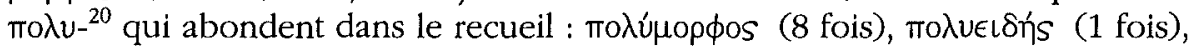

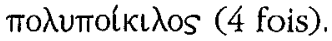

Polymorphes et omniprésents, les dieux des Hymnes sont aussi caractérisés par une fonction cosmique qui fait d'eux l'origine et les garants de l'ordre du monde. On a vu dans le cas de l'hymne à Héraklès comment le motif traditionnel des travaux est étroitement mêlé à une dimension

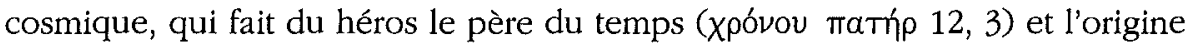

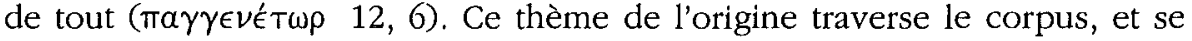

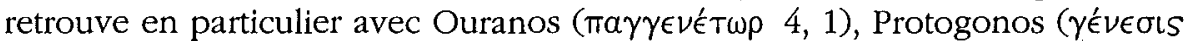

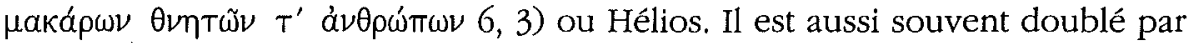
un motif selon lequel les dieux assurent la rotation du monde : ce sont les images de $\delta i ́ n \eta(4,4 ; 7,4 ; 40,15)$ et pópßos $(4,4 ; 8,7)$. Enfin les dieux des Hymnes sont souvent liés à la lumière. Traditionnel dans le cas de divinités comme Hélios, ce motif est aussi présent dans des situations plus surprenantes. La Nuit des Hymnes, par exemple, brille de tous feux, à la fois

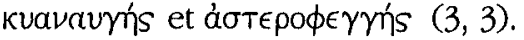

A côté de ce motif cosmique et de ses différentes manifestations, l'élément dionysiaque constitue un autre thème unificateur du corpus. Une première indication est donnée par la présence massive de Dionysos, auquel sont dédiés, sous des noms variés, huit hymnes du recueil. D'autre part, le

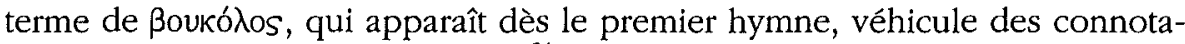
tions dionysiaques bien marquées ${ }^{21}$. Des motifs dionysiaques parcourent les hymnes à Dionysos, Perséphone et Silène, mais apparaissent aussi dans des contextes moins attendus. Ainsi des Néréides, qui deviennent prêtresses des rites de Bakkhos et Perséphone :

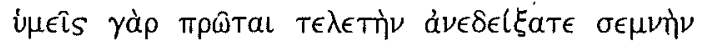

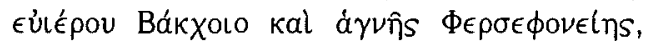

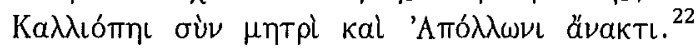

Liées à ce thèmes dionysiaque, on remarque quelques allusions à des mythes dits " orphiques » car présents dans d'autres textes transmis sous le nom d'Orphée. $M$. West ${ }^{23}$ a ainsi relevé certains échos entre les Rhapsodies et les Hymnes, comme l'union mystique de Zeus et Déméter dont naît Perséphone $(29,5-7)$, celle de Zeus et Perséphone dont naît Dionysos (30, 6-7), le fait que les Titans soient les ancêtres de l'humanité (37, 2-6), les Euménides

20 Sur les composés en mo $\lambda v$ - comme convention du genre de l'hymne, voir F. Porta, Greek Ritual Utterances and the Litungical Style, Diss. Harvard, 1999, p. 230-232.

21 Sur le ßoukónos comme titre parmi les fidèles de Dionysos, voir R. MErkelbach, Die Hirten des Dionysos: Die Dionysos-Mysterien der römiscben Kaiserzeit und der bukoliscbe Roman des Longus, Stuttgart, 1988, p. 61-62.

22

$\mathrm{OH}, 24,10-12$.

23 M. West, The Orpbic Poems, Oxford, 1986, p. 252-253. 
filles de Zeus et de Perséphone $(70,2-3$ et 29,6$)$ et l'importance de Protogonos. Ces allusions restent peu nombreuses, et on est frappé par l'absence apparente de mythes « orphiques » importants, comme le démembrement de Dionysos Zagreus ${ }^{24}$.

Ces motifs communs, dont on n'a dressé ici qu'une esquisse, contribuent à donner du panthéon des Hymnes orpbiques une image relativement homogène, justifiant le qualificatif de syncrétique. Cependant, les dieux des Hymnes ne sont pas complètement indifférenciés. Le fonctionnement des hymnes comme prière conduit en effet à les caractériser de façon plus précise, en utilisant des épithètes qui ont un rôle spécifique par rapport à la demande finale.

S'il n'a pas encore été possible d'établir précisément les conditions dans lesquelles étaient utilisés les Hymnes orphiques ${ }^{25}$, il n'en reste pas moins que ces textes se présentent formellement comme des prières : l'invocation au dieu, qui occupe la majeure partie de chaque hymne, est suivie d'une demande, énoncée sur un à trois vers et qui conclut le texte ${ }^{26}$. La prière finale commence souvent par une demande générale du type "écoute-moi » ( $\kappa \lambda \nu \tilde{\theta}$ $\mu o v$ ) ou « viens » ("̈ $\rho \chi \epsilon \sigma \theta a \mathrm{l}$ ou $\mu \mathrm{o} \lambda \epsilon \tilde{\mathrm{L} \nu}$ à l'impératif, optatif ou infinitif), parfois

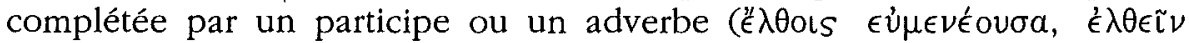

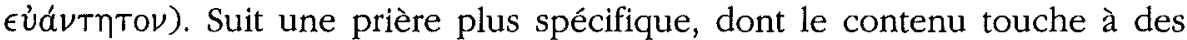
domaines variés. On peut pour la commodité de l'exposé y distinguer trois grandes catégories, tout en soulignant que les hymnes mêlent souvent plusieurs demandes relevant de champs différents.

Une partie importante des demandes concerne le rite lui-même; on implore la divinité d'être présente lors des mystères $(\tau \epsilon \lambda \epsilon \tau a l)^{27}$ ou aux côtés des initiés ${ }^{28}$. Parfois c'est simplement la présence bienveillante de la divinité qui est demandée ${ }^{29}$. Dans un cas, on implore le dieu (Dionysos Liknites) de recevoir favorablement les sacrifices (lepá 46, 8); dans un autre, il est demandé à Mnémosyne d'aider les initiés à se souvenir du rite sacré ( $\epsilon$ ưtépos

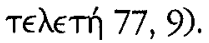

24 N'entre pas dans le présent propos la question difficile de l'« orphisme » des Hymnes. Voir en particulier à ce sujet RudhardT, l.c. (n. 1), p. 269.

25 Depuis les études d'O. Kern (l.c. [n. 2], p. 87 sq. et "Das Demeterheiligtum von Pergamon und die orphischen Hymnen", Hermes 46 [1911], p. 431-436), un consensus s'est établi pour voir dans les Hymnes orpbiques des textes rituels, peut-être utilisés par une communauté d'Asie mineure (Pergame) au ${ }^{\mathrm{e}}{ }^{\mathrm{e}}$ s. ap. J.-C. $C f$. C. Calame, "Orphik. Orphische Dichtung", Der neue Pauly (à paraître). L'hypothèse de textes littéraires n'est cependant pas entièrement rejetée. Cf. Hunsucker, o.c. (n. 1), p. 53-58.

26 On retrouve ici la structure normale des prières. Cf. PoRTA, o.c. (n. 20), p. 160-162.

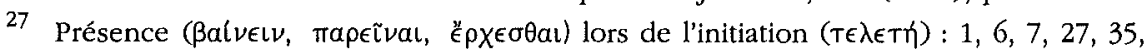
$43,49,51,53,79$; lors des sacrifices $(l \in \rho \alpha)$ : 54 .

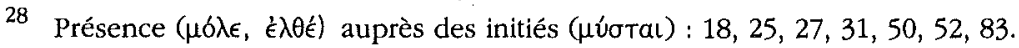

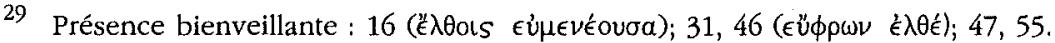


A côté de ces prières qui portent sur le bon fonctionnement du rite, les demandes concernent aussi des aspects plus généraux de la vie. Certains hymnes implorent une vie sainte, douce ou noble ${ }^{30}$. Une quinzaine d'hymnes demandent paix, santé et prospérité, parfois accompagnées de justice ${ }^{31}$; d'autres demandent simplement l'aide ${ }^{32}$ ou la faveur ${ }^{33}$ du dieu. Une dizaine d'hymnes implorent une fin de vie heureuse ${ }^{34}$. Les demandes portent aussi sur des qualités morales ${ }^{35}$ ou intellectuelles ${ }^{36}$.

Enfin le quotidien n'est pas oublié, au travers de prières qui demandent des conditions météorologiques favorables à la navigation ou à l'agriculture ${ }^{37}$ ou des récoltes abondantes ${ }^{38}$. L'hymne à Eileithyia demande un accouchement facile $(2,13-4)$, tandis que l'aide de Poséidon (17), Leucothée (74) ou Palémon (75) est requise pour la navigation. Les prières apotropaïques reflètent en négatif les mêmes sortes de préoccupations que les demandes positives. Il s'agit d'écarter les ennuis quotidiens ${ }^{39}$, mais aussi la souffrance, les maladies et la mort ${ }^{40}$, et les maladies de l'âme: folie, cauchemars, peurs, pensées mauvaises ${ }^{41}$.

Les bénéficiaires de la demande ne sont pas toujours précisés, mais lorsqu'ils le sont, il s'agit la plupart du temps des initiés $(\mu u ́ \sigma \tau a \mathrm{l})^{42}$. Dans

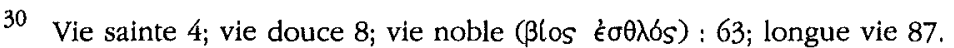

31 Paix, santé et prospérité : 10,15,17, 19, 23, 32; paix et richesses : 14; prospérité $(\delta \lambda \beta 0 S): 24,60$; paix, justice, prospérité et santé : 40, 84; justice 62; paix 65; prospérité et richesses 72 .

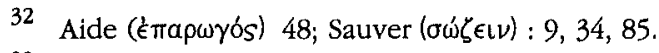

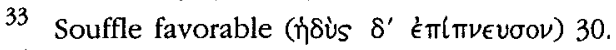

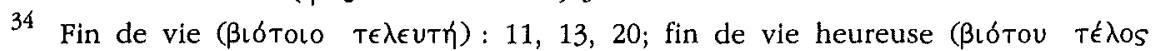

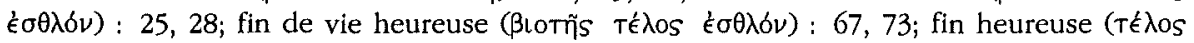

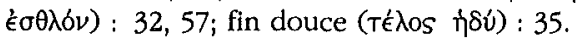

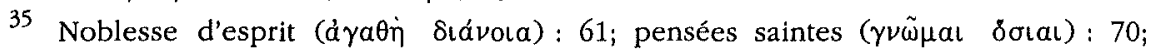
pensées droites 86 ; bienveillance 44,71 ; joie $(\gamma \tilde{\eta} \theta 0 s) 45$; justice $(\mu \nu \eta \dot{\mu \eta} \sigma \in$ o, demandé à Nomos) 64.

36 Vie intellectuelle fructueuse 19; émulation qui amène la gloire 76.

37 Ciel calme 5; pluie 21, 51, 82; vent pour navigation : $22,74,81$; beau temps 80 ; lumière 78.

38 Récoltes fertiles $26,29,36,38,40,43,53,56$.

39 Tremblements de terre 23 ; mauvaise mer 75 ; nuages et pluie 80.

40 Maladies et mort 12 ; maladies et souffrances 36 ; maladies 68 ; souffrances ( $\kappa \dot{r} \delta \in \alpha$ ) 73.

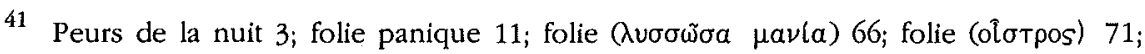
impiété et mort 14; colère 37, 39; фavtaólal 39; impulsions basses et étrangères 58; pensées impures 61; souffrance 59; méchanceté humaine 63; discorde et souffrances morales 65; signes qui conduisent au mal 86.

42 Initiés ( $\mu$ v́oтal) : 8, 18, 24, 34, 36, 44, 50, 56, $57,58,59,60,61,71,74,75,76,77,83$;

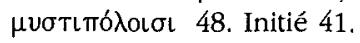


quelques cas on prie pour le ou les nouveaux initiés ${ }^{43}$; deux hymnes ( 1 et 31 ) demandent une faveur pour le ßovkó $\lambda$ os; deux autres ont une portée plus universelle : l'hymne 63 (à Dikè) concerne les hommes et tous les être vivants, tandis que l'hymne 45 (à Dionysos Bassareus Trieterikos) prie pour tous. En dehors de l'hymne 59 aux Moires, la première personne est absente de la demande finale, si ce n'est à travers le pronom dans la formule $\kappa \lambda \tilde{u} \theta \underline{\imath} \mu$ ov.

Énonçant une demande souvent dirigée vers un bénéficiaire particulier, chaque hymne de la collection peut donc s'analyser comme une instance de communication, un acte d'énonciation qui met en rapport un locuteur (la personne qui récite l'hymne) et un destinataire (la divinité à qui l'hymne est adressé) dont il s'agit d'obtenir une faveur (fonction conative). Que des hymnes constituent une argumentation chargée de convaincre la divinité d'accorder une faveur n'est pas propre aux Hymnes orphiques. J. Bremer ${ }^{44}$ a montré que ce fonctionnement caractérise la plupart des hymnes de culte, et a distingué plusieurs modes d'argumentation (da quia dedi, da ut dem, da quia dedisti, da quia boc dare tuum est) pouvant être mis en oeuvre. L'importance argumentative des mots est cependant particulièrement sensible dans les Hymnes orphiques. Le lien entre qualité de l'hymne et obtention de la faveur est d'ailleurs souligné dans le dernier vers de l'hymne à Aphrodite :

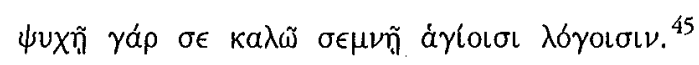

L'énoncé de la demande y est suivi d'un hexamètre final qui souligne les raisons $(\gamma a ́ \rho)$ pour lesquelles la prière doit être exaucée : parce que l'orant invoque la divinité d'une âme pieuse $(\psi v \times n ̃$ $\sigma \epsilon \mu \nu \eta ̣ ̂)$ et en utilisant des termes

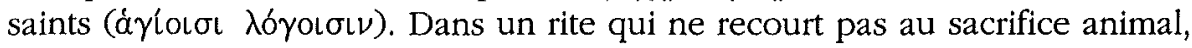
le fidèle ne possède pas d'autre moyen de convaincre la divinité que les mots eux-mêmes, en dehors d'encens et de quelques libations de lait : $\theta u \sigma i a ı \sigma$ kal

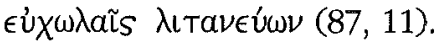

Si la plupart des hymnes cultuels ont pour fonction de convaincre la divinité, on considère habituellement que c'est surtout la partie intermédiaire de l'hymne qui remplit ce rôle. J. Bremer ${ }^{46}$ a même montré qu'il est justifié d'appeler cette partie « argument », remplaçant ainsi le qualificatif de « pars epica » proposé par C. Ausfeld ${ }^{47}$ mais adapté surtout au modèle des Hymnes homériques. Puisque les Hymnes orphiques ne présentent pas réellement de partie intermédiaire entre l'invocation et la prière, l'argument chargé de convaincre la divinité ne peut se trouver que dans l'invocation. Comme le

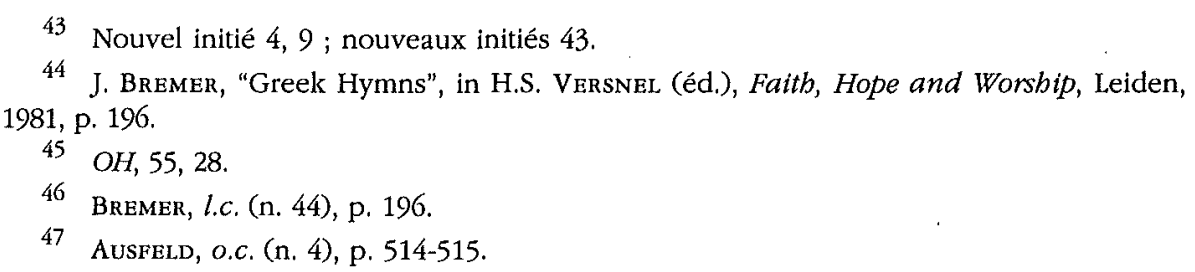


suggère J. Rudhardt, à travers la série des noms, épithètes et propositions relatives qui développent l'invocation initiale, l'orant veut « éveiller l'attention de la divinité invoquée, mobiliser tous ses pouvoirs, pour mieux assurer l'efficacité de sa propre démarche ${ }^{48}$. Autrement dit, ce sont les épithètes qui sont chargées d'assurer la réussite de la prière finale.

Ce lien entre épithètes et demande finale est illustré par la continuité formelle qui unit invocation et demande. Le verbe conjugué qui ouvre

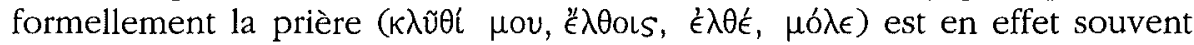
suivi de participes ou propositions participiales qui modifient et précisent la façon dont la divinité est priée de venir ou d'écouter. On retrouve dans la prière les épithètes de l'invocation, à tel point qu'il est parfois difficile de distinguer l'une de l'autre, comme dans l'hymne 54 à Silène, Satyros et aux Bacchantes : la prière, d'ailleurs inhabituellement longue, y est précisée par une suite d'épithètes et propositions participiales (54, 7-11).

Parmi les formes argumentatives distinguées par Bremer, c'est plutôt.le $d a$ quia boc dare tuum est qui est sous-jacent dans les Hymnes orpbiques. Ceux-ci s'attachent en effet à montrer que la demande finale relève du champ d'action de la divinité tel qu'il est dépeint aux travers des épithètes. Le dernier vers de l'hymne à Prothyraia-Eileithyia est révélateur de ce mode de fonctionnement :

kal $\sigma \omega \hat{\zeta}$,

Par le jeu étymologique, l'énoncé de la prière $(\sigma \omega \tilde{\zeta} \epsilon)$ est directement mis en relation avec une qualité qui participe de la nature ( $\left.\not \phi v_{S}\right)$ de la déesse.

Ce principe du da quia boc dare tuum est justifie une partie des épithètes appliquées à chaque divinité. Eileithyia-Prothyraia,' à laquelle il est demandé son aide dans une naissance, est invoquée par des épithètes qui soulignent sa fonction particulière de déesse des accouchements :

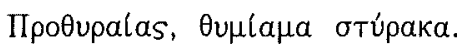

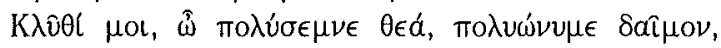

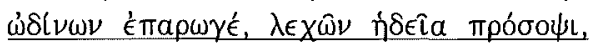

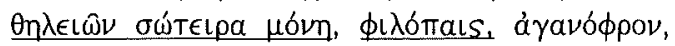

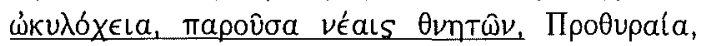

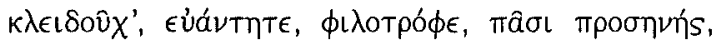

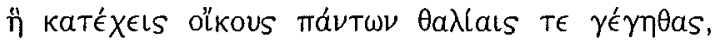

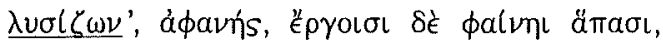

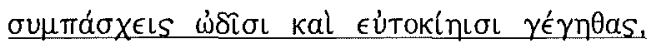

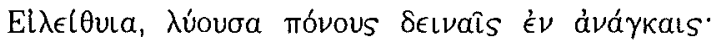

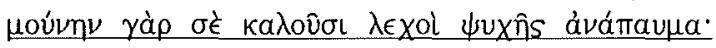




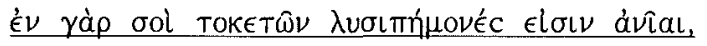

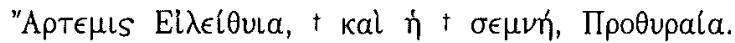

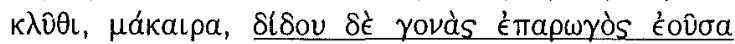

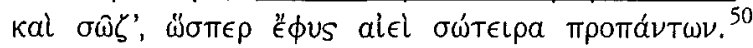

Cette correspondance entre épithètes et demande finale est traduite, sur le plan du vocabulaire, par l'utilisation de mots identiques, formés sur la même racine ou synonymes dans l'invocation et dans la prière. Ainsi Hipta, à laquelle il est demandé d'être présente aux mystères ("̈ 7) est décrite dans l'invocation comme exultant dans les mystères du saint

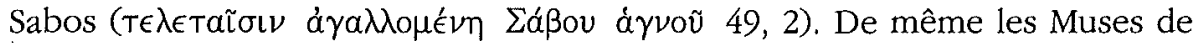
l'hymne 51, auxquelles il est demandé la pluie, sont présentées tout au long

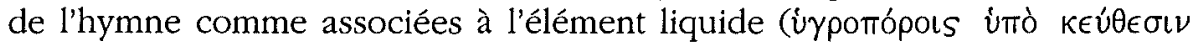

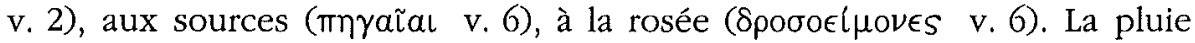

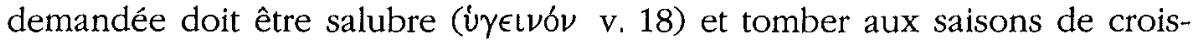

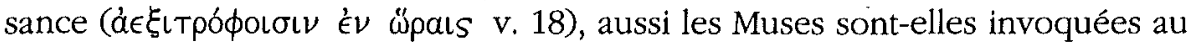

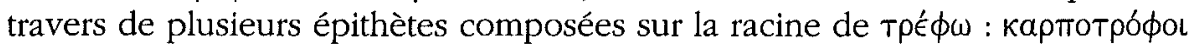

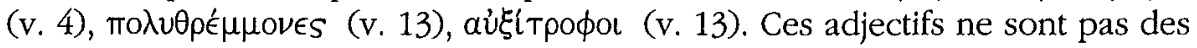
épithètes traditionnelles des Muses; ils ne leur sont pas propres non plus, puisqu'on les retrouve appliqués à d'autres divinités : les Nuées sont égale-

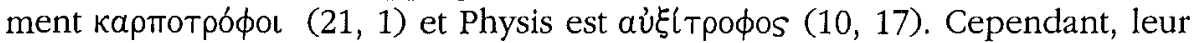
emploi dans l'hymne 51 est tout à fait pertinent dans l'optique de la demande finale. Il apparaît ainsi que les épithètes non traditionnelles utilisées dans les Hymnes orphiques obéissent à un double critère de sélection : d'une part, de grands motifs communs, qui contribuent au syncrétisme du recueil; d'autre part, la préoccupation de donner de chaque divinité une image en rapport avec la demande spécifique qui lui est adressée.

Le fonctionnement des Hymnes orphiques comme prière permet peutêtre aussi d'expliquer leur caractéristique la plus frappante, ces listes d'épithètes juxtaposées qui en constituent la majeure partie. De façon générale, l'utilisation d'épithètes dans les prières a souvent été mise en rapport avec un pouvoir quasi magique des noms: l'accumulation d'épithètes serait un moyen de forcer la divinité à accorder la faveur demandée. Ainsi Burkert avance-t-il que « im Kult ist es Aufgabe des Betenden, durch Beinamen den Gott gleichsam einzukreisen und den rechten, treffenden Namen zu finden ${ }^{51}$. Cette idée a également été appliquée aux Hymnes orphiques ${ }^{52}$, sur

$50 \mathrm{OH}, 2$ (Eileithyia). C'est moi qui souligne les épithètes et propositions relatives et participiales qui caractérisent Eileithyia dans sa fonction de déesse des accouchements.

51 W. BURKERT, Griecbiscbe Religion der arcbaiscben und klassiscben Epocbe, Stuttgart, 1977, p. 285. Voir aussi la discussion de S. PulleyN, Prayer in Greek Religion, Oxford, 1997, p. 98-115.

52 Voir en particulier O. GrupPe, "Orphische Hymnen", in W.H. Roscher (éd.), Ausfürliches Lexicon der griecbiscben und römiscben Mytbologie III, Leipzig, 1897-1909. 
la base de leur ressemblance avec certaines invocations des papyrus magiques.

L'accumulation d'épithètes juxtaposées a certes un effet incantatoire que l'on pourrait mettre en relation avec certaines pratiques magiques. Le ton est donné par le premier hymne du recueil, l'hymne à Hécate, dont chaque hexamètre de l'invocation commence par un adjectif choriambique (si l'on admet la diérèse au vers 4). Tout au long du recueil, la tendance est à remplir l'hexamètre avec quatre ou cinq adjectifs, parfois selon la même répartition des dactyles et spondées :

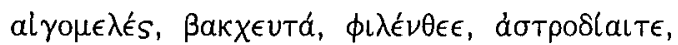

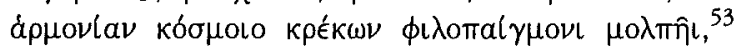

Un autre élément de régularité rythmique tient à la structure anaphorique de certaines invocations, comme dans le premier vers de l'hymne à Eileithyia :

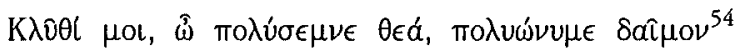

Ces phénomènes métriques et rythmiques sont doublés de jeux sur les sonorités, au travers de répétitions, polyptotes et antithèses qui donnent aux hymnes leur unité. Ainsi les épithètes de l'hymne à Dionysos sont liées entre elles par la répétition d'un même élément, d'un composé à l'autre :

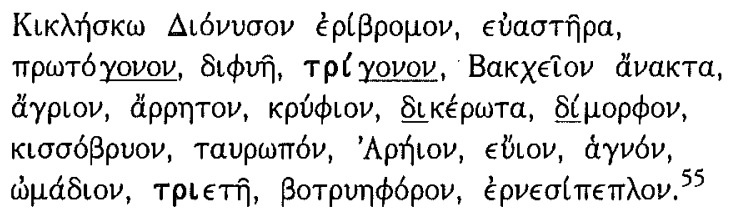

L'accumulation d'épithètes joue donc de répétitions et de rythmes pour faire des Hymnes orpbiques, selon l'expression de C. Calame, des « chants incantatoires $"$ ". Par certains aspects, le langage devient presque le générateur du texte. Il n'est cependant pas nécessaire de mettre ces phénomènes musicaux en rapport avec des pratiques magiques. Le lien avec la musique et le chant est caractéristique du genre de l'hymne, comme l'attestent les partitions des hymnes delphiques à Apollon étudiées par Annie Bélis ${ }^{57}$ et d'autres témoignages ${ }^{58}$. D'autre part, les épithètes utilisées dans les Hymnes

$53 \mathrm{OH}, 11,5-6$ (Pan).

54 OH, $2,1$.

$55 \mathrm{OH}, 30,1-5$.

56 Calame, l.c. (n. 25).

57 A. BÉLIs, Corpus des inscriptions de Delphes, t. 3 : Les hymnes à Apollon, Paris, 1992.

58 PAus., V, 15, 10 et II, 7, 4; LSAM 33a.21 $=S I G^{3}$ 695 = I. Magn. $100 \mathrm{Kern}$; Neue Inscbr. Ephesos XII, 25. 
orphiques ne semblent pas aller dans le sens de pratique de magie coercitive. Bon nombre d'entre elles sont moins des noms que des adjectifs, qui ne peuvent se substituer au théonyme puisqu'ils s'appliquent à plusieurs divinités. Il semble qu'il faille donc aller chercher dans d'autres directions les conséquences de l'accumulation d'épithètes.

Un premier effet est celui de concentration, de raccourci. J. Rudhardt ${ }^{59}$ a montré que les épithètes ne doivent pas toutes être mises sur le même plan et que l'une peut déterminer l'autre au lieu d'être directement reliée au nom du

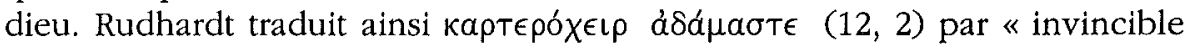
grâce à la puissance de tes bras » et elapıvaì $\lambda \in t \mu \omega \nu$ ládes $(43,3)$ par « qui, au printemps, fréquentez les prairies humides ». Ainsi « le groupe épithétique » forme-t-il « l'équivalent d'une proposition » et « une syntaxe » est-elle «latente sous la parataxe ${ }^{60}$. Les épithètes concentrent en quelques mots ce qui pourrait être exprimé par une proposition entière.

Cette idée de concentration est confirmée par l'analyse d'autres textes qui recourent aux listes d'épithètes. Deux des quatre hymnes à Isis transmis sous le nom d'Isidore (datés du début du premier siècle av. J.-C.) s'ouvrent par des invocations constituées d'épithètes juxtaposées, souvent composées, dont les ressemblances thématiques et formelles avec les Hymnes orphiques ont été notées par Véra Vanderlip ${ }^{61}$. Ainsi le premier hymne :

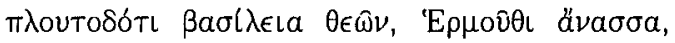

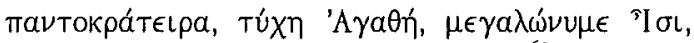

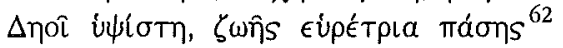

et le troisième :

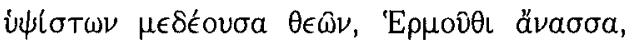

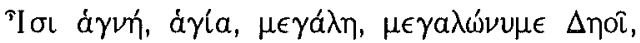

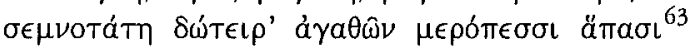

Cependant, dans les Hymnes à Isis, cette invocation initiale est suivie d'une partie plus narrative, riche en verbes 'conjugués, qui justifie les

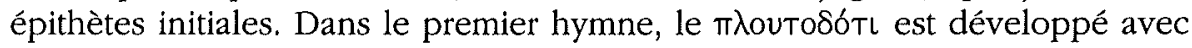
l'évocation des dons d'Isis aux hommes (lois, technai; fertilité grâce aux crues du Nil, 1, 4-13). De même, le $\mu \epsilon \gamma a \lambda \omega ́ v v \mu \epsilon$ est largement explicité au travers de l'énumération des divers noms donnés à Isis par les différents peuples (1, 14-24). Dans les Hymnes à Isis, les épithètes initiales fonctionnent

\footnotetext{
59 RudHARDT, l.c. (n. 1), p. 264-269.

60 RuDHARDT, l.c. (n. 1), p. 267.

61 V. VANDerlip, The Four Greek Hymns of Isidomis and the Cult of Isis, Toronto, 1972 (American Studies in Papyrology, 12).

62 IsIDORE, Hymnes, 1, 1-3.

63 IsIDORE, Hymnes, 3, 1-3.
} 
donc comme un prélude qui concentre les grands thèmes de l'hymne. Cette comparaison suggère que les listes d'épithètes sont perçues comme le moyen d'aller le plus rapidement possible à l'essentiel, d'évoquer de façon concise ce qui peut être illustré ensuite par plusieurs vers.

Parallèlement à cet effet de concision, on peut remarquer que la conséquence de l'accumulation d'épithètes est de donner des dieux une image qui relève de la description, par opposition à la narration. Le petit nombre de formes verbales personnelles est une conséquence frappante de l'accumulation des épithètes. Pour ne prendre qu'un exemple, je n'en relève que trois

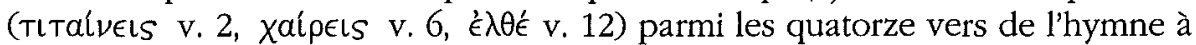
Rhéa (14).

Cette absence est compensée d'une part par la présence d'adjectifs composés qui comprennent souvent un élément verbal (adjectifs déverbaux), d'autre part par des participes présents, souvent du verbe être. Ainsi dans l'hymne aux Astres, les vers 5 et 6 contiennent trois adjectifs formés sur un

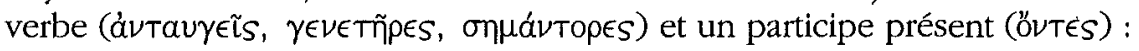

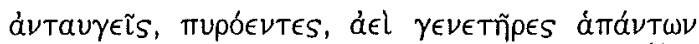

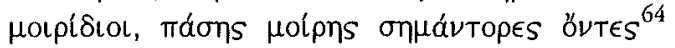

L'effet de la substantivation, cependant, est remarquable en ce qu'elle supprime les nuances temporelles et modales habituellement véhiculées par le verbe conjugué et donne de la divinité une image hors du temps, atemporelle et amodale. Le verbe sous-entendu est le verbe être, conjugué à un éternel présent.

Cette idée que les listes d'épithètes visent à décrire la divinité, à dire de façon concise ce que l'on pourrait presque appeler l'« être » ou l'« essence » du dieu invoqué, est confirmée par le rôle de listes semblables dans certains des oracles de Claros $^{65}$. L'oracle inscrit sur un portail d'Oinoanda ${ }^{66}$, également cité dans la Théosophie de Tübingen ${ }^{67}$ et par Lactance ${ }^{68}$, se présente comme une réponse oraculaire qui, pour dire ce qu'est le dieu, a justement recours à une liste d'épithètes :

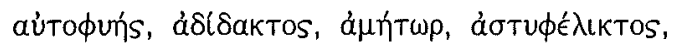

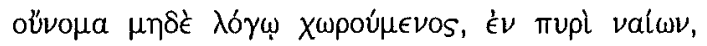

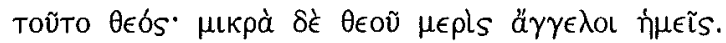

\footnotetext{
$64 \mathrm{OH}, 7,5-6$.
}

65 Sur ces oracles, voir T. ROBINson, Theological Oracles and the Sanctuaries of Claros and Didyma, Diss. Harvard, 1981 et R. Merkelbach, J. Stauber, "Die Orakel des Apollon von Klaros", EA 27 (1996), p. 1-52.

66 Sur l'oracle d'Oinoanda, voir Robert, l.c. (n. 5), dont je suis le texte.

67 Theos. 13. Cf. H. ERBSE, Fragmente griechischer Theosophien, Hamburg, 1941 (Hamburger Arbeiten zur Altertumswissenschaft, Band 4).

68 LAC'., Div. Inst. 1, 7, 1. 
La Théosophie donne de cet oracle une version plus longue, qui s'ouvre sur le verbe être, 'ै $\sigma \theta^{\prime}$, se poursuit par treize vers comportant des verbes conjugués au présent et se termine par les trois vers inscrits à Oinoanda. Enfin Lactance précise que l'oracle comportait vingt-et-un vers, mais n'en cite que trois qui sont précisément ceux de l'inscription, et que Lactance appelle le principium de l'oracle. Puisque le témoignage de la Théosopbie place la liste d'épithètes à la fin de l'oracle, il semble qu'il faille comprendre, avec Buresch $^{69}$, que principium signifie ici « la somme, le total ». Dans l'oracle d'Oinoanda, la liste d'épithètes représente donc le principium, la somme, de

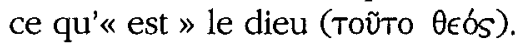

Un fonctionnement semblable est observé dans un oracle d'Apollon cité par Eusèbe ${ }^{70}$ et provenant peut-être aussi de Claros $^{71}$. Comme un fidèle avait

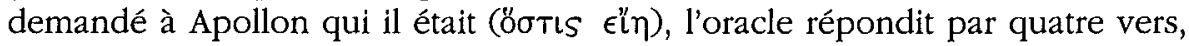
dont le premier est composé d'une liste de noms propres, le deuxième d'une proposition adjectivale, le troisième d'une proposition participiale et le dernier de deux groupes nominaux :

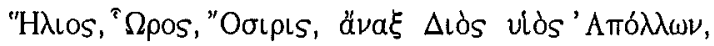

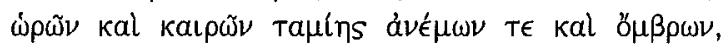

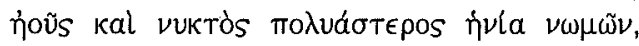

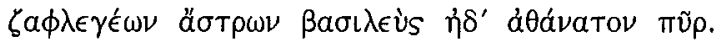

Ici aussi, une structure semblable à celle des Hymnes orphiques est utilisée pour définir la nature ou l'essence d'un dieu. Il convient certes de distinguer le contexte religieux des oracles de Claros et celui des Hymnes. Les oracles participent d'une conception fortement hénothéiste, tandis que les Hymnes, malgré leur tendance au syncrétisme, ne véhiculent pas la notion d'un dieu suprême. La ressemblance de stucture reste cependant frappante, et suggère que les listes d'épithètes des Hymnes visent à décrire ce que les fidèles considéraient comme la nature ou l'essence de la divinité invoquée.

La sélection des épithètes ainsi que leur organisation sous forme de listes sont donc révélatrices du fonctionnement des Hymnes orphiques et des conceptions qu'ils véhiculent. Parallèlement aux épithètes traditionnelles et individuelles, les dieux y sont glorifiés au travers d'épithètes communes qui mettent en valeur leur fonction cosmique et leur rôle dans la mythologie dionysiaque. Ces grands thèmes traversent la collection et donnent au panthéon des Hymnes une certaine homogénéité, justifiant le qualificatif de

69 K. Buresch, Klaros, Untersuchungen zum Orakelwesen des späteren Altertums, Leipzig, 1889. Pour une discussion de cet usage de principium, cf. RoBinson (o.c. [n. 65], p. 80-99), qui d'ailleurs réfute l'hypothèse de Buresch et suit l'émendation praecipuum proposée par Wolff.

70 Eus., $P E$ III, $15,125 \mathrm{c}-\mathrm{d}$.

71 Cf. RoBinson, o.c. (n. 65), p. 339-341. 
syncrétisme. Il ne faudrait pas cependant en conclure que les divinités y sont indifférenciées et confondues. Au sein de chaque hymne, un bon nombre d'épithètes s'attachent à caractériser le dieu de façon plus spécifique et à montrer que la demande finale relève de ses pouvoirs. À ce souci d'efficacité de la prière est aussi liée la technique de juxtaposition des épithètes. La comparaison avec les hymnes d'Isidore à Isis et certains oracles de Claros suggère en effet que ces listes étaient perçues comme un moyen de définir la nature de la divinité invoquée, et donc de mobiliser toute sa puissance.

Sélection paradigmatique comme arrangement syntagmatique font donc ressortir le double statut des Hymnes orpbiques, à la fois textes littéraires et cultuels. S'ils empruntent à la tradition littéraire un fonds de vocabulaire et le goût de l'allusion savante, ils se rattachent à la pratique rituelle par le souci d'assurer l'efficacité de la prière en mobilisant l'essentiel des pouvoirs du dieu, en fonction du contenu spécifique de la demande finale. À partir de ce double statut, on peut rêver à ce qu'étaient les fidèles qui pratiquaient ces textes. Leurs prières demandent une vie sainte, des qualités morales, mais aussi la richesse, la santé ou des conditions favorables à l'agriculture et à la navigation. Peut-on alors voir dans les $\mu$ úrtal des hommes cultivés, des lettrés, soucieux aussi du quotidien?

Marianne HoPMAN-Govers

Harvard University

Paris IV-Sorbonne 